\title{
Factorization Method and Solution of the Non-Central Modified Kratzer Potential
}

\author{
J. SADEGHI* \\ Sciences Faculty, Department of Physics, Mazandaran University \\ P.O. Box 47415-416, Babolsar, Iran
}

(Received July 2, 2007)

In this paper, we consider the non-central modified Kratzer potential. We tried to connect the corresponding Schrödinger equation to the associated Laguerre and Jacobi equation. For this purpose the factorization information from associated function was employed to solve the corresponding equations. It leads us to have some raising and lowering operators which are first-order equations. These operators help us to obtain all quantum states and energy spectrum for different $n$ and $m$. The obtained results show that the degeneracy of the second quantum number $(m)$ is completely removed.

PACS numbers: 03.65.--w, 11.10.Lm, 11.30.Na, 12.39.St

\section{Introduction}

The Kratzer or modified Kratzer-type potentials [1], which we consider in this paper, have played an important role in the history of the molecular structure and interactions [2]. This potential offered one of the most important exact models of atomic and molecular physics and quantum chemistry. It may be applied to energy spectrum for the $\mathrm{CO}$ diatomic molecule with different quantum numbers. Also the analytical solution of the radial Schrödinger equation is of high importance in non-relativistic quantum mechanics since the wave function contains all the necessary information to describe a quantum system fully. As we know the quasi-exact solution for the radial Schrödinger equation within a given potential is given by Ref. [3]. In the spherical coordinates, the Schrödinger equation with the non-central modified Kratzer potential is

$$
\begin{gathered}
-\frac{\hbar^{2}}{2 \mu}\left[\frac{1}{r^{2}} \frac{\partial}{\partial r}\left(r^{2} \frac{\partial}{\partial r}\right)+\frac{1}{r^{2} \sin \theta} \frac{\partial}{\partial \theta}\left(\sin \theta \frac{\partial}{\partial \theta}\right)+\frac{1}{r^{2} \sin ^{2} \theta} \frac{\partial^{2}}{\partial \varphi^{2}}\right] \psi \\
+\left[D\left(\frac{r-a}{r}\right)^{2}+\frac{\beta^{\prime}}{r^{2} \sin ^{2} \theta}+\frac{\gamma \cos \theta}{r^{2} \sin ^{2} \theta}\right] \psi=E \psi,
\end{gathered}
$$

\footnotetext{
*e-mail: pouriya@ipm.ir
} 
where the non-central modified Kratzer potential is

$$
V(r, \theta)=D\left(\frac{r-a}{r}\right)^{2}+\frac{\beta^{\prime}}{r^{2} \sin ^{2} \theta}+\frac{\gamma \cos \theta}{r^{2} \sin ^{2} \theta},
$$

and $D$ is the dissociation energy, $a$ is the equilibrium internuclear separation, $\beta^{\prime}$ and $\gamma$ are strictly positive constants. The first term of this potential is the modified Kratzer potential, the second and third terms are the angle dependent parts.

If the spherical total wave function as $\psi(r, \theta, \varphi)=R(r) Y(\theta, \varphi)$ is inserted into Eq. (1), the wave equation for the non-central modified Kratzer potential is separated into variables and the following equations are obtained:

$$
\frac{\mathrm{d}^{2} R}{\mathrm{~d} r^{2}}+\frac{2}{r} \frac{\mathrm{d} R}{\mathrm{~d} r}+\frac{2 \mu}{\hbar^{2}}\left[E-\frac{D(r-a)^{2}}{r^{2}}\right] R-\frac{\lambda}{r^{2}} R=0,
$$

and

$$
\begin{aligned}
& \frac{\mathrm{d}^{2} \Theta(\theta)}{\mathrm{d} \theta^{2}}+\cot \theta \frac{\mathrm{d} \Theta(\theta)}{\mathrm{d} \theta}+\left(\lambda-\frac{m^{2}}{\sin ^{2} \theta}-\frac{2 m}{\hbar^{2}} \frac{\beta^{\prime}+\gamma \cos \theta}{\sin ^{2} \theta}\right) \Theta(\theta)=0, \\
& \frac{\mathrm{d}^{2} \Phi(\varphi)}{\mathrm{d} \varphi^{2}}+m^{\prime 2} \Phi(\varphi)=0
\end{aligned}
$$

where

$$
Y(\theta, \varphi)=\Theta(\theta) \Phi(\varphi) .
$$

First we are going to discuss the radial part of the Schrödinger equation corresponding to the Kratzer potential.

\section{Radial part of the Schrödinger equation}

The radial part of the Schrödinger equation given by Eq. (2) can be written as

$$
r \frac{\mathrm{d}^{2} R}{\mathrm{~d} r^{2}}+2 \frac{\mathrm{d} R}{\mathrm{~d} r}+\frac{2 \mu}{\hbar^{2} r}\left[(E-D) r^{2}+2 D a r-\left(D a^{2}+\frac{\lambda \hbar^{2}}{2 \mu}\right)\right] R=0 .
$$

We choose the following variable:

$$
R(r)=U(r) L(r),
$$

so we have

$$
\begin{aligned}
& r L^{\prime \prime}(r)+\left(2 r \frac{U^{\prime}}{U}+2\right) L^{\prime}(r)+\left[r \frac{U^{\prime \prime}}{U}+\frac{2 U^{\prime}}{U}+\frac{2 \mu}{\hbar^{2}}(E-D) r+\frac{4 D \mu a}{\hbar^{2}}\right. \\
& \left.-\frac{2 \mu}{\hbar^{2}}\left(D a^{2}+\frac{\lambda \hbar^{2}}{\mu}\right) \frac{1}{r}\right] R=0 .
\end{aligned}
$$

In order to obtain the parameters ( $D$ and $\lambda$ ), eigenfunction and eigenvalue for the non-central Kratzer potential we compare Eq. (7) with the following associated Laguerre differential equation [4-6]:

$$
\begin{aligned}
& r L_{n, m}^{\prime \prime}(\alpha, \beta)+(1+\alpha-\beta r) L_{n, m}^{\prime(\alpha, \beta)}(r) \\
& \quad+\left[\left(n-\frac{m}{2}\right) \beta-\frac{m}{2}\left(\alpha+\frac{m}{2}\right) \frac{1}{r}\right] L_{n, m}^{(\alpha, \beta)}(r)=0,
\end{aligned}
$$


so we obtain the $U(r)$ and $D$,

$$
U(r)=\mathrm{e}^{-\beta r / 2} r^{(\alpha-1) / 2},
$$

and

$$
D=\frac{\hbar^{2}}{4 \mu a}[(n-m / 2) \beta+(\alpha+\beta) / 2] .
$$

From Eqs. (6) and (9) one can obtain the corresponding eigenfunction

$$
R(r)=\mathrm{e}^{-\beta r / 2} r^{(\alpha-1) / 2} L_{n, m}^{\alpha, \beta}(r) .
$$

In that case the exact energy eigenvalues of the radial part of the Schrödinger equation with the non-central Kratzer potential are derived as

$$
E_{n, m}=\frac{\hbar^{2}}{4 \mu a}\left[\left(n-\frac{m}{2}\right) \beta+\frac{\alpha+\beta}{2}-\frac{\beta^{2}}{2}\right]
$$

also the $\lambda$ is separation constant which is obtained by

$$
\begin{aligned}
\lambda= & \frac{1}{2}\left[\frac{m}{2}\left(\alpha+\frac{m}{2}\right)+\frac{\alpha-1}{2}+\frac{(\alpha-1)^{2}}{4}-\frac{1}{2 a}\left(n-\frac{m}{2}\right) \beta\right. \\
& \left.-\frac{1}{4 a}(\alpha+\beta)\right] .
\end{aligned}
$$

We note here that the solution associated Laguerre equation in the Rodrigues representation is

$$
L_{n, m}^{\alpha, \beta}(x)=\frac{a_{n, m}(\alpha, \beta)}{r^{\alpha+\frac{m}{2}} \mathrm{e}^{-\beta r}}\left(\frac{\mathrm{d}}{\mathrm{d} x}\right)^{n-m}\left(r^{n+\alpha} \mathrm{e}^{-\beta r}\right),
$$

in which $a_{n, m}(\alpha, \beta)$ is the normalization coefficient, and is also obtained by

$$
a_{n, m}(\alpha, \beta)=(-1)^{m} \sqrt{\frac{\beta^{\alpha+m+1}}{\Gamma(n-m+1) \Gamma(n+\alpha+1)}} .
$$

Here we also discuss the raising and lowering operators which correspond to the radial part of the Kratzer potential. Therefore, we can factorize the associated Laguerre differential equation with respect to the parameters $n$ and $m$ as follows:

$$
\begin{aligned}
& A_{n, m}^{+}(r) A_{n, m}^{-}(r) L_{n, m}^{(\alpha, \beta)}(r)=(n-m)(n+\alpha) L_{n, m}^{(\alpha, \beta)}(r), \\
& A_{n, m}^{-}(r) A_{n, m}^{+}(r) L_{n-1, m}^{(\alpha, \beta)}(r)=(n-m)(n+\alpha) L_{n-1, m}^{(\alpha, \beta)}(r),
\end{aligned}
$$

where the differential operators as functions of parameters $n$ and $m$ are, respectively

$$
A_{n, m}^{+}(r)=r \frac{\mathrm{d}}{\mathrm{d} r}-\beta r+\frac{2 n+2 \alpha-m}{2}, \quad A_{n, m}^{-}(r)=-r \frac{\mathrm{d}}{\mathrm{d} r}+\frac{2 n-m}{2} .
$$

Let us note that the shape in variance Eq. (16) can also be written as the raising and lowering relation

$$
\begin{aligned}
& A_{n, m}^{+}(r) L_{n-1, m}^{(\alpha, \beta)}(r)=\sqrt{(n-m)(n+\alpha)} L_{n, m}^{(\alpha, \beta)}(r), \\
& A_{n, m}^{-}(r) L_{n, m}^{(\alpha, \beta)}(r)=\sqrt{(n-m)(n+\alpha)} L_{n-1, m}^{(\alpha, \beta)}(r) .
\end{aligned}
$$


Therefore, we obtain the raising and lowering operators for the radial part of the Kratzer potential. These operators help us to have bound states for that system.

\section{Polar angle part of the Schrödinger equation}

We may also derive the eigenvalues and eigenfunctions of the polar angle part of the Schrödinger equation similar to the solution of the radial part. By introducing a new variable $x=\cos \theta$, we can write Eq. (3) as follows:

$$
\frac{\mathrm{d}^{2} \Theta(x)}{\mathrm{d} x^{2}}-\frac{2 x}{1-x^{2}} \frac{\mathrm{d} \Theta(x)}{\mathrm{d} x}+\frac{\lambda\left(1-x^{2}\right)-m^{\prime 2}-\frac{2 m^{\prime}}{\hbar^{2}}\left(\beta^{\prime}+\gamma x\right)}{\left(1-x^{2}\right)^{2}} \Theta(x)=0 .
$$

With the choice of the following variable:

$$
\Theta(x)=U(x) P(x),
$$

we rewrite Eq. (19) as follows:

$$
\begin{aligned}
& \left(1-x^{2}\right) P^{\prime \prime}(x)+\left[\frac{2 U^{\prime}\left(1-x^{2}\right)}{U}-2 x\right] P^{\prime}(x)+\left[\left(1-x^{2}\right) \frac{U^{\prime \prime}}{U}-2 x \frac{U^{\prime}}{U}+\lambda\right. \\
& \left.-\frac{m^{\prime 2}-\frac{2 m^{\prime}}{\hbar^{2}}\left(\beta^{\prime}+\gamma x\right)}{1-x^{2}}\right] P(x)=0 .
\end{aligned}
$$

Also here, in order to obtain the parameters, eigenfunction, and eigenvalue for the non-central Kratzer potential we compare Eq. (21) with the following associated Jacobi differential equation [7]:

$$
\begin{aligned}
& \left(1-x^{2}\right) P_{n, m}^{\prime \prime}(x)-[\alpha-\beta+(\alpha+\beta+2) x] P_{n, m}^{\prime} \\
& \quad+\left[n(\alpha+\beta+n+1)-\frac{m[\alpha+\beta+m+(\alpha-\beta)] x}{1-x^{2}}\right] P_{n, m}(x)=0
\end{aligned}
$$

where

$$
m=\frac{2 m^{\prime}}{\hbar^{2}}-\frac{\alpha+\beta}{2} .
$$

Therefore, for the energy spectrum we obtain

where

$$
E_{n, m^{\prime}}=\frac{\hbar^{2}}{4 \mu a}\left[\left(n-\frac{m^{\prime}}{\hbar^{2}}+\frac{(\alpha+\beta)}{4}\right) \beta+\frac{\alpha+\beta}{2}-\frac{\beta^{2}}{2}\right],
$$

$$
\alpha+\beta=\beta^{\prime}, \quad \alpha-\beta=\gamma .
$$

The corresponding wave functions are found as

$$
\Theta_{n, m}(x)=\left[\frac{\beta-\alpha}{2} \int\left(\frac{\mathrm{d} x}{1-x^{2}}\right)-\frac{\alpha+\beta}{2} \int \frac{x}{\left(1-x^{2}\right)} \mathrm{d} x\right] P_{n, m}^{(\alpha, \beta)}(x) .
$$

The associated Jacobi functions $P_{n, m}^{(\alpha, \beta)}(x)$ as the solution of the differential equation have the following Rodrigues representation:

$$
P_{n, m}^{(\alpha, \beta)}(x)=\frac{a_{n, m}(\alpha, \beta)}{(1-x)^{\alpha+\frac{m}{2}}(1+x)^{\beta+\frac{m}{2}}}\left(\frac{\mathrm{d}}{\mathrm{d} x}\right)^{n-m}\left[(1-x)^{\alpha+n}(1+x)^{\beta+n}\right] .
$$

Now we are going to discuss the raising and lowering operators corresponding to 
the non-central Kratzer polar angle part of the Schrödinger equation. As mentioned in Refs. [8, 4, 7], we can write the associated Jacobi differential Eq. (21) as the following:

$$
\begin{aligned}
& A_{n, m}^{+}(x) A_{n, m}^{-}(x) P_{n, m}^{\left(\beta^{\prime}, \gamma\right)}(x)=B_{n, m} P_{n, m}^{\left(\beta^{\prime}, \gamma\right)}(x), \\
& A_{n, m}^{-}(x) A_{n, m}^{+}(x) P_{n-1, m}^{\left(\beta^{\prime}, \gamma\right)}(x)=B_{n, m} P_{n-1, m}^{\left(\beta^{\prime}, \gamma\right)}(x),
\end{aligned}
$$

where

$$
\begin{aligned}
& B_{n, m}=\frac{(n-m)\left(\beta^{\prime}+\gamma+2 n\right)\left(\beta^{\prime}+m+n\right)}{\left(\beta^{\prime}+2 n\right)^{2}}, \\
& A_{n, m}^{+}(x)=\left(1-x^{2}\right) \frac{\mathrm{d}}{\mathrm{d} x}-\left(\beta^{\prime}+n\right) x-\gamma \frac{\beta^{\prime}+n+m}{\beta^{\prime}+2 n}, \\
& A_{n, m}^{-}(x)=-\left(1-x^{2}\right) \frac{\mathrm{d}}{\mathrm{d} x}-n x+\gamma \frac{n-m}{\beta^{\prime}+2 n} .
\end{aligned}
$$

In the case of shape invariance with respect to $m$ we have

$$
\begin{aligned}
& A_{m}^{+}(x) A_{m}^{-}(x) P_{n, m}^{\left(\beta^{\prime}, \gamma\right)}(x)=C_{n, m} P_{n, m}^{\left(\beta^{\prime}, \gamma\right)}(x), \\
& A_{m}^{-}(x) A_{m}^{+}(x) P_{n, m-1}^{\left(\beta^{\prime}, \gamma\right)}(x)=C_{n, m} P_{n, m-1}^{\left(\beta^{\prime}, \gamma\right)}(x),
\end{aligned}
$$

where

$$
C(n, m)=(n-m+1)\left(\beta^{\prime}+n+m\right)
$$

and

$$
\begin{aligned}
& A_{m}^{+}=\sqrt{1-x^{2}} \frac{\mathrm{d}}{\mathrm{d} x}+\frac{m-1}{\sqrt{1-x^{2}}} x, \\
& A_{m}^{-}=-\sqrt{1-x^{2}} \frac{\mathrm{d}}{\mathrm{d} x}+\frac{\gamma+\beta^{\prime}+m}{\sqrt{1-x^{2}}} x .
\end{aligned}
$$

Also, these operators help us to discuss the bound states for the corresponding potential. In two cases we can see that the energy spectrum is independent of parameters $\beta^{\prime}$ and $\gamma$.

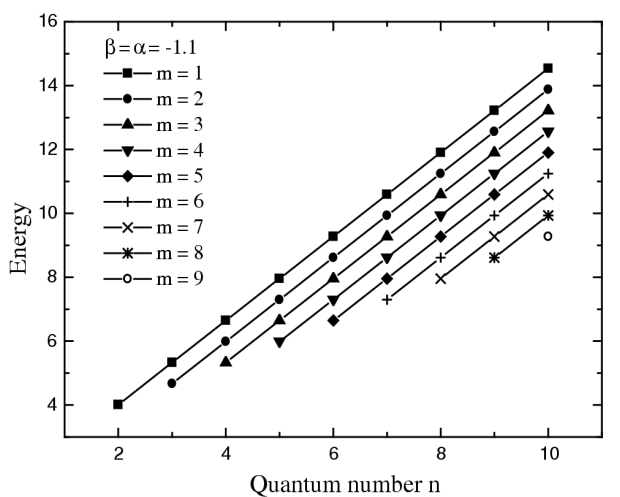

Fig. 1. The energy spectrum of solving the radial part of the Schrödinger equation. $\beta$ and $\alpha$ are arbitrary numbers. 


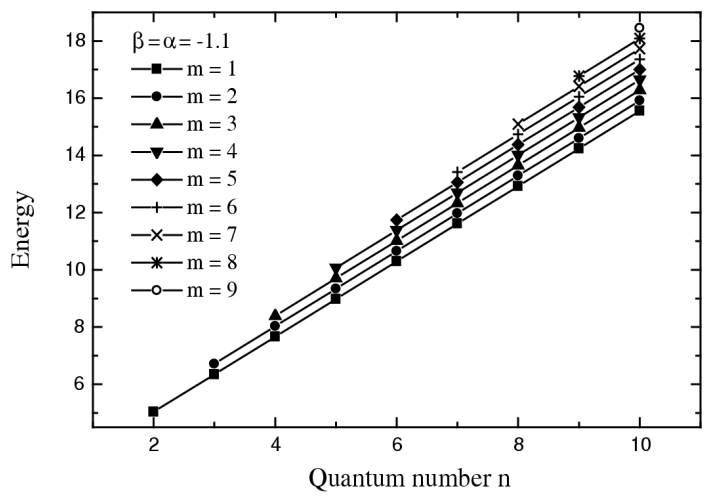

Fig. 2. The energy spectrum of solving the angular part of the Schrödinger equation. $\beta$ and $\alpha$ are arbitrary numbers.

In order to show the effect of the modified Kratzer potential on the energy spectrum, the obtained energy spectrum of radial and angular part from solving Schrödinger equation are illustrated in Fig. 1 and Fig. 2 as a function of quantum numbers $n$ and $m$, respectively.

\section{Conclusion}

We have shown that in a systematic approach the energy spectrum and the corresponding eigenfunctions of the Schrödinger equation with non-central potentials can be easily obtained by using the method presented in this study.

As we see in Figs. 1 and 2 the degeneracy of the second quantum number is completely removed also for the special values of $n$ and $m(m \leq n-1)$. In Fig. 1 the variation of energy spectrum decreases but in Fig. 2 this energy increases. These results may be applied in future to the CO-like diatomic molecule for different quantum numbers.

\section{References}

[1] A. Kratzer, Z. Phys. 3, 289 (1920).

[2] R.J. Le Roy, R.B. Bernstein, J. Chem. Phys. 52, 3869 (1970).

[3] C.L. Pekeris, Phys. Rev. 45, 98 (1934).

[4] M.A. Jafarizadeh, H. Fakhri, Ann. Phys. (New York) 262, 260 (1998).

[5] A.F. Nikiforov, V.B. Uvarov, Special Functions of Mathematical Physics: A Unified Introduction with Applications, Birkhäuser, Basel 1988.

[6] J. Sadeghi, Eur. Phys. J. B 50, 453 (2006).

[7] H. Fakhri, J. Sadeghi, Mod. Phys. Lett. A 19, 615 (2004).

[8] M.A. Jafarizadeh, H. Fakhri, Phys. Lett. A 230, 164 (1997). 